\title{
Effects of functional electrical stimulation on dysphagia caused by radiation therapy in patients with nasopharyngeal carcinoma
}

\author{
Pei-Hung Lin • Tzu-Yu Hsiao • Yeun-Chung Chang • \\ Lai-Lei Ting • Wen-Shiang Chen • Su-Chiu Chen • \\ Tyng-Guey Wang
}

Received: 29 September 2009 / Accepted: 11 November 2009 /Published online: 29 November 2009

(C) Springer-Verlag 2009

\begin{abstract}
Purpose This study aimed to assess the effectiveness of functional electrical stimulation on the swallowing function of irradiated nasopharyngeal carcinoma patients with dysphagia.
\end{abstract}

\section{P.-H. Lin}

Department of Speech and Hearing Disorders and Sciences,

National Taipei College of Nursing,

Taipei, Taiwan, Republic of China

\section{T.-Y. Hsiao}

Department of Otolaryngology,

National Taiwan University Hospital,

School of Medicine, National Taiwan University,

Taipei, Taiwan, Republic of China

\section{Y.-C. Chang}

Department of Medical Imaging,

National Taiwan University Hospital,

School of Medicine, National Taiwan University,

Taipei, Taiwan, Republic of China

\section{L.-L. Ting}

Department of Oncology, National Taiwan University Hospital, School of Medicine, National Taiwan University,

Taipei, Taiwan, Republic of China

W.-S. Chen $\cdot$ T.-G. Wang $(\bowtie)$

Department of Physical Medicine and Rehabilitation,

National Taiwan University Hospital,

School of Medicine, National Taiwan University,

7 Chung-Shan South Road,

Taipei 100 Taiwan, Republic of China

e-mail: tgw@ntu.edu.tw

\section{S.-C. Chen}

Department of Health Care Management, National Taipei College of Nursing,

Taipei, Taiwan, Republic of China
Materials and methods Twenty nasopharyngeal carcinoma subjects with dysphagia were divided into a functional electrical stimulation (FES) and a home rehabilitation program (HRP) group. Each subject completed a quality of life questionnaire on swallowing and a videofluoroscopic study at the commencement and the end of the procedures. The FES group received functional electrical stimulation of the supra-hyoid muscles over 15 sessions. The HRP group performed self-swallowing exercises at home. The evaluation parameters included the quality of life questionnaire scores, the penetration-aspiration scale (PAS), the movement of the hyoid bone, and the amount of pyriform sinuses stasis.

Results Most swallowing outcomes of the FES group improved after FES. The quality of life score $(p=0.003)$, the duration of the movement of thin barium through the hyoid $(p=0.001)$, the moving speed of paste barium through the hyoid ( $p=0.028$ ), and the pyriform sinus stasis area of the paste barium $(p=0.026)$ reached significant difference in the FES group. Most swallowing outcomes did not improve in the HRP group. The degree of improvement in the movement speed of the hyoid bone in the thin barium $(p=0.018)$ and the PAS of the paste barium $(p=0.016)$ were statistically significantly greater in the FES group than in the HRP group. Conclusion FES will improve the swallowing function of NPC patients with dysphagia and bring about better quality of life.

Keywords Nasopharyngeal carcinoma - Radiotherapy . Functional electrical stimulation - Dysphagia - Quality of life

\section{Introduction}

Nasopharyngeal carcinoma (NPC) is a common disease among Southeast Asia [1]. Radiotherapy (RT) is the most 
common primary treatment for NPC with good outcomes reported, but complications frequently arise. These are well documented and include hearing loss, xerostomia, trismus, local soft tissue fibrosis, and dysphagia [2]. Xerostomia, dysphagia, and hearing loss, which feature prominently among these, most negatively impact quality of life (QoL) [3]. Dysphagia also increases the risk of aspiration, which may cause death [4].

The prevalence of dysphagia following RT ranges from $27 \%$ to $80 \%$ of NPC patients depending on the assessment methods, the definition of swallowing impairment, radiation dosage, and the duration of post-RT $[5,6]$. Its main features include delayed pharyngeal transit time, decreased pharyngeal contraction, incomplete hyoid bone elevation, increased stasis of oral mucosa, and aberrant upper esophageal sphincter (UES) function [5]. Aspiration pneumonia often arises from incomplete hyoid bone elevation and aberrant UES function [6]. It has been suggested that the opening of the UES relates to elevation of the hyoid bone [7].

Studies have shown that dysphagia in head and neck cancer patients following RT may be reduced by compensatory skills such as posture adjustment, changes to the characteristics of food consistency, and muscle strengthening [8-13]. This further reduces the probability of aspiration. Swallowing exercises include tongue holding exercises, resistance exercises, effortful swallowing, and Shaker's exercise. But once dysphagia has set in, these exercises cannot strengthen muscles. No studies have yet explored possibilities for strengthening swallowing-related muscles after RT to effectively treat dysphagia once it has already developed $[12,13]$.

Functional electrical stimulation (FES) has recently been found to improve swallowing function in patients with dysphagia following cerebral vascular accidents. By stimulating the supra-hyoid muscle group, FES improves the elevation of the hyoid bone and the larynx system, which restores the function of the protective mechanism of the airway and the opening of the UES [16, 21]. However, these findings have not been analyzed in detail [14-21]. Moreover, no studies have yet investigated how FES may treat dysphagia in post-RT NPC patients.

This study therefore aimed to explore this technique as a preliminary to further investigation into FES as a feasible treatment for this debilitating side effect of NPC.

\section{Materials and methods}

Participants

The inclusion criteria were: (1) subjects were diagnosed with NPC; (2) subjects had completed RT over a year before this study; and (3) subjects' swallowing function was less than level 6 on the Dysphagia Outcome and Severity Scale (DOSS) [22]. Excluded from the study were subjects who (1) suffered a neurological or degenerative disease; (2) had received oral-pharynx tissue resection; and (3) had epilepsy or an implanted cardiac pacemaker. Twenty subjects participated in this study. They were assigned into the FES group and home rehabilitation program (HRP) group by the method of sex stratified randomization, and were matched by DOSS score within 1 unit in order to compare two groups under the same sex proportion and similar DOSS score pairs (Table 1). The study was approved by the Ethics Committee of the National Taiwan University Hospital. All participants gave their informed consent.

Assessment

The QoL questionnaire and videofluoroscopic study (VFSS) examination were performed before and after treatment. The QoL questionnaire is the Chinese translation version of the M.D. Anderson Dysphagia Inventory (MDADI) [23] and was performed by a speech-language pathologist (SLP). The translated questionnaire was reviewed and revised for

Table 1 Patient characteristics

\begin{tabular}{lll}
\hline Characteristics & FES & HRP \\
\hline $\begin{array}{l}\text { Total patient number } \\
\text { Age (years) }\end{array}$ & 10 & 10 \\
$\quad$ Mean \pm SD & $52.3 \pm 7.4$ & $56.1 \pm 11.8$ \\
$\quad$ Range & $37-64$ & $40-72$ \\
Sex (male/female) & $3: 2$ & $3: 2$ \\
AJCC stage, IIB/III/IVA/NA, number & $3 / 4 / 3 / 0$ & $0 / 6 / 3 / 1$ \\
Radiotherapy: number of sessions & & \\
One session (70 Gy) & 8 & 9 \\
$\quad$ Two sessions(140 Gy) & 2 & 1 \\
Interval ${ }^{\mathrm{a}}$ (years) & & \\
Mean \pm SD & $9.1 \pm 4.0$ & $8.1 \pm 9.5$ \\
Range & $3-18$ & $1-35$ \\
DOSS, score & & \\
Mean \pm SD & $3.8 \pm 1.5$ & $3.8 \pm 1.2$ \\
Range & $1-6$ & $1-5$ \\
Median & 4 & 4 \\
MDADI, total score & & \\
Mean \pm SD & $45.1 \pm 10.8$ & $57.5 \pm 11.3$ \\
Range & $34-67$ & $45-72$ \\
\hline
\end{tabular}

FES functional electrical stimulation, HRP home rehabilitation program, AJCC American Joint Committee on Cancer (5th edn, 1997), NA unknown, DOSS dysphagia outcome and severity scale, MDADI M.D. Anderson Dysphagia Inventory

${ }^{\mathrm{a}}$ Number of years between finishing radiotherapy and participation in this study 
appropriate content validity by one professor in speechlanguage pathology, two clinical SLPs, and one researcher in public health. The questionnaire has 20 items, each with one to five points to indicate "strongly disagree" to "strongly agree." Each subject's overall score indicates the swallowing aspect of their QoL, a score of 100 being the most satisfactory. Standardized VFSS was meanwhile performed using a remote-controlled fluoroscope (KOX-850, Toshiba Corp., Tokyo, Japan; RSZ-2000, Shimadzu Corp., Kyoto, Japan) equipped with a high-resolution Super-VHS recorder (BR 1200, JVC, Japan). The videotape recorder had a frame rate of 30 frames per second and could display real-time dynamic images and frame-by-frame static images. Patients sat on a chair customized for both lateral and frontal anterior-posterior views (VESS chair, Vess Chairs Inc., Milwaukee, WI, USA). Each patient swallowed $5 \mathrm{ml}$ each of thin and paste barium sulfate (E-Z-HD, E-Z-EM, Inc., Westbury, NY, USA) bolus. The thin barium sulfate comprised a suspension of $340 \mathrm{~g}$ E-Z-HD and $65 \mathrm{ml}$ of water. The paste form of the barium sulfate formula was prepared by adding an extra $12 \mathrm{ml} \mathrm{E-Z-HD} \mathrm{powder} \mathrm{to} \mathrm{a}$ $15-\mathrm{ml}$ standard thin barium preparation.

\section{Treatment}

Each FES subject received VitalStim ${ }^{\circledR}$ therapy (Chattanooga Group, TN, USA) from a certified and experienced SLP. The therapy comprised fifteen 60-min sessions, one to three times per week. The FES paired electrodes were placed above the hyoid bone and between the hyoid bone and thyroid cartilage (Fig. 1) to stimulate the supra-hyoid muscles. The current frequency and on-time were set at $80 \mathrm{~Hz}$ and $700 \mu$ s, and the intensity was adjusted to each subject's maximum tolerance. All subjects made continuous swallowing attempts during the treatment. Where necessary, water was provided to assist swallowing.

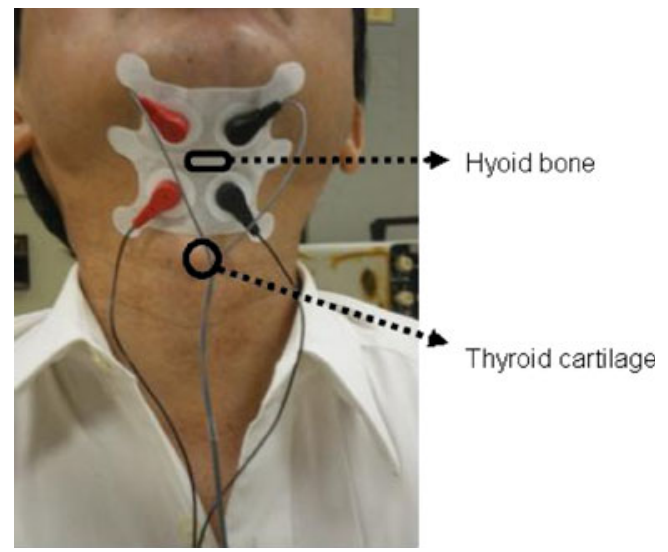

Fig. 1 Electrodes of the VitalStim ${ }^{\circledR}$ were applied above the hyoid bone and between the hyoid bone and thyroid cartilage
Table 2 Therapeutic exercises in home rehabilitation program group

\begin{tabular}{|c|c|}
\hline Exercise characteristics & Description \\
\hline $\begin{array}{l}\text { Range of motion } \\
\text { exercise }\end{array}$ & $\begin{array}{l}\text { Hold lips/tongue maximum extent of } \\
\text { movement for } 5 \mathrm{~s}\end{array}$ \\
\hline Resistance exercise & $\begin{array}{l}\text { Push lips/tongue against a tongue blade } \\
\text { for } 5 \mathrm{~s}\end{array}$ \\
\hline Tongue-hold exercise & $\begin{array}{l}\text { Swallow while keeping tip of tongue } \\
\text { nipped softly between the teeth }\end{array}$ \\
\hline Effortful swallow & $\begin{array}{l}\text { Swallow hard to improve tongue base } \\
\text { retraction }\end{array}$ \\
\hline Shaker exercise & $\begin{array}{l}\text { Lie down and hold head to see toe for } \\
1 \mathrm{~min} \text {, followed by } 1 \text {-min rest, and three } \\
\text { times per session; Repeat the action } \\
\text { quickly for } 1 \mathrm{~s} \text {, followed by } 1 \text {-s rest, } \\
\text { and } 30 \text { times per session }\end{array}$ \\
\hline
\end{tabular}

The ten HRP subjects were instructed by the SLP to perform twice-daily strengthening exercises and repeat each item more than ten times during each session (Table 2). We telephoned these subjects every 2 weeks to encourage them to maintain their exercise program.

\section{Data measurement}

A parameter of the VFSS assessment was the penetrationaspiration scale (PAS), which divides the condition of aspiration into eight levels. The higher the level, the more severe the aspiration condition [24]. The oral transit time (OTT) is the period from when the tongue started to pass the bolus posterior to the top of the pharynx. The pharyngeal transit time (PTT) is the time from arrival of the bolus head at the intersection of the jaw and the root of the tongue until the bolus tail exited the pharynx and passed into the UES. The pharyngeal delay time (PDT) is the time between the start of PTT and the moment when the hyoid bone began to move upward and forward [25]. The displacement of the hyoid bone was defined as that from the resting position to the highest position. Therefore, the anterior displacement of the hyoid bone was indicated by its translation along the $X$-axis and the superior displacement its translation along the $Y$-axis. The duration of the hyoid bone displacement was defined as the time spent moving from the resting position to the highest position. The velocity was defined as the displacement divided by the duration. The areas of barium sulfate stasis in the valleculae and the pyriform sinus were also calculated. The PAS, OTT, PPT, and PDT were evaluated through dynamic (real time) and static images (frame by frame) at a sampling rate of $30 \mathrm{~Hz}$. To measure the hyoid bone-related parameters, the evaluator used two VFSS images: one with the hyoid bone at the resting position before swallowing (Fig. 2a) and the other with the hyoid bone at the highest position during swallowing (Fig. 2b). A 
Fig. 2 Measurement of the displacement of the hyoid bone. a Resting position of the hyoid bone before swallowing. b Highest position of the hyoid bone during swallowing. The hyoid (solid circle) and the third vertebra of the $\mathrm{C}$-spine (hollow circle) are also indicated
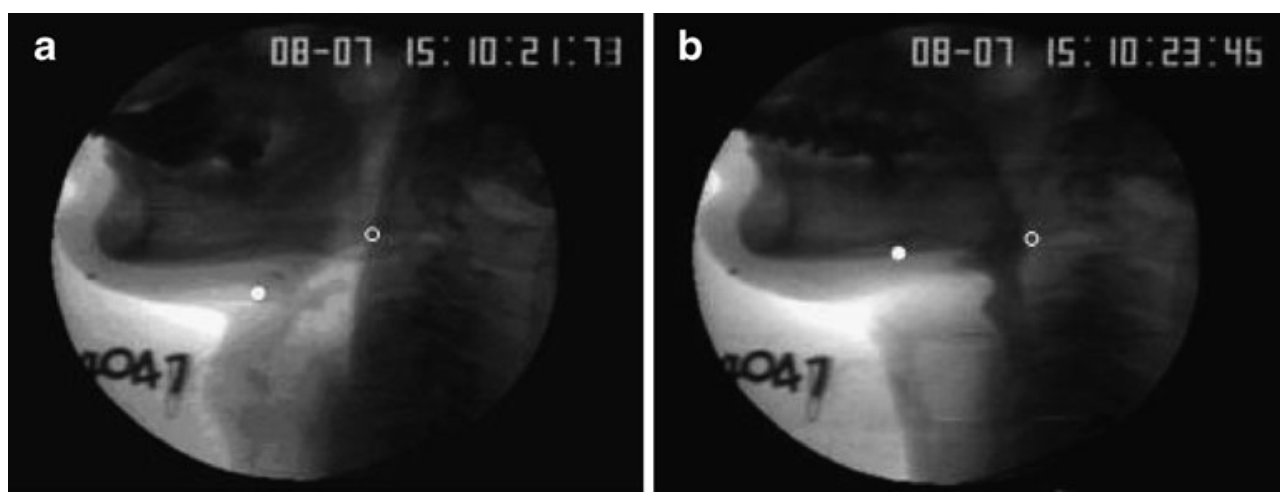

2.5-cm paper clip was used as a scale and the anterior upper margin of third vertebra of the $\mathrm{C}$-spine as the origin point $(0,0)$ to measure the displacement of the hyoid bone from the resting position to the highest position and the superior and anterior displacements. To calculate the bolus stasis areas of the pharynx, the evaluator used the VFSS images of the bolus stasis in the valleculae (Fig. 3a) and pyriform sinus (Fig. 3b), segmented the stasis areas by curves, and then calculated the segmented areas using the paper clip as a scale. Both the hyoid-related parameters and stasis areas of the pharynx were analyzed with MATLAB ${ }^{\circledR} 7.5$ (MathWorks, USA).

The consistency of the assessment was evaluated before the experiment based on the VFSS images of ten dysphagia patients by two SLP who were not aware of each other's results. The assessment items were the PAS, OTT, PTT, and PDT and the displacement, anterior and superior displacements, displacement duration and velocity of the hyoid bone, as well as the bolus stasis areas in the valleculae and pyriform sinus.

\section{Statistical analysis}

Measurement reliability was analyzed using intra-class correlation coefficients (ICC). An ICC $<0.4$ indicated poor correlation, while an ICC $>0.7$ indicated excellent correlation. An ICC between 0.4 and 0.7 indicated an acceptable correlation. Comparisons of the outcome measures before and after therapy for both groups and comparisons of the changes of the outcome measures after therapy were analyzed using the paired $t$ test (two-tailed) with SPSS 15.0 (LEAD Technologies, USA). A $p$ value of $<0.05$ was considered statistically significant.

\section{Results}

Reliability of measurement

The values of intra-ICC and of inter-ICC were greater than 0.7 in all the measurement parameters in this work.

Comparisons of the FES group before and after therapy

In the FES group, the MDADI score increased from $45.1 \pm$ 10.8 to $53.1 \pm 10.7(p=0.003)$. The VFSS results show that although the PAS for swallowing the thin and the paste solution decreased from $3.8 \pm 2.9$ to $2.9 \pm 2.8(p=0.253)$ and from $3.5 \pm 2.5$ to $3.0 \pm 2.7 \quad(p=0.138)$, respectively, no statistically significant improvement was found. The temporal parameters showed no significant changes for either the thin or the paste barium. Following therapy, the hyoid bone displacements showed no significant changes during swallowing, but the velocity increased significantly from $1.8 \pm 1.2$ to $2.6 \pm 1.4 \mathrm{~cm} / \mathrm{s}(p=0.001)$ for the thin
Fig. 3 Measurement of the area of bolus stasis by marking with dotted lines the areas of the stasis of the vallecula (a) and stasis of the pyriform sinuses (b)
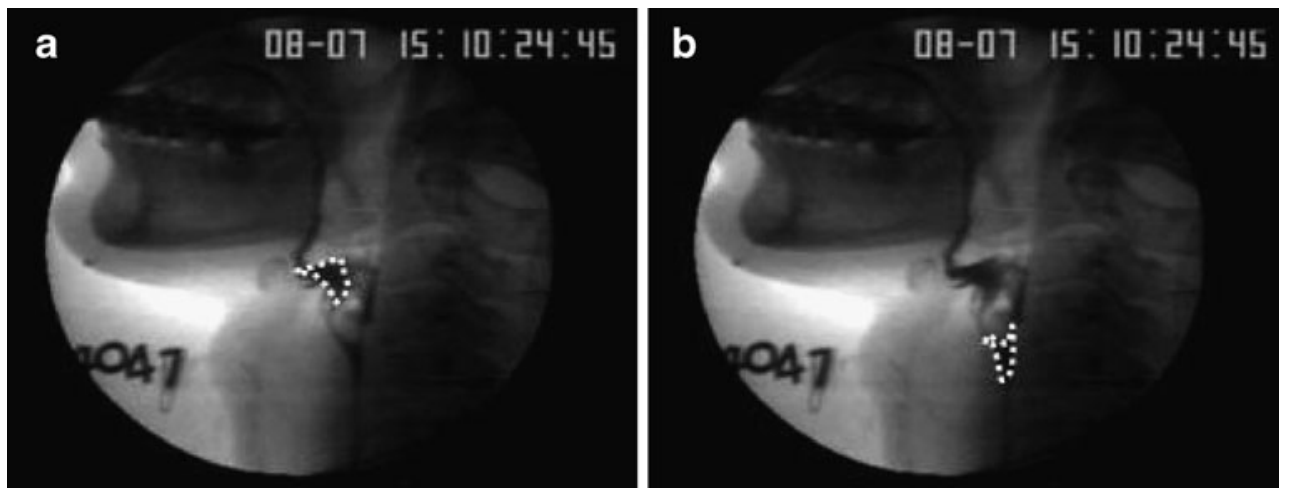
Table 3 Pre- and post-treatment in FES group
$* p<0.05$, paired $t$ test

\begin{tabular}{|c|c|c|c|}
\hline Measurements & Pre-, mean \pm SD & Post-, mean \pm SD & $p$ \\
\hline M.D. Anderson Dysphagia Inventory, total score & $45.1 \pm 10.8$ & $53.1 \pm 10.7$ & $0.003^{*}$ \\
\hline \multicolumn{4}{|l|}{$5 \mathrm{ml}$ thin $(n=10)$} \\
\hline Penetration-aspiration scale, score & $3.8 \pm 2.9$ & $2.9 \pm 2.8$ & 0.253 \\
\hline Oral transit time $(\mathrm{s})$ & $0.9 \pm 1.1$ & $0.7 \pm 0.5$ & 0.344 \\
\hline Pharyngeal transit time (s) & $2.2 \pm 2.6$ & $3.7 \pm 4.8$ & 0.300 \\
\hline Pharyngeal delay time (s) & $0.6 \pm 0.9$ & $0.4 \pm 0.7$ & 0.733 \\
\hline Maximum displacement of hyoid bone $(\mathrm{cm})$ & $1.2 \pm 0.4$ & $1.2 \pm 0.6$ & 0.561 \\
\hline Forward displacement of hyoid bone (cm) & $0.6 \pm 0.4$ & $0.8 \pm 0.4$ & 0.472 \\
\hline Upward displacement of hyoid bone (cm) & $0.9 \pm 0.5$ & $0.9 \pm 0.6$ & 0.916 \\
\hline Displacement duration of hyoid bone (s) & $1.0 \pm 0.7$ & $0.6 \pm 0.4$ & $0.010^{*}$ \\
\hline Displacement velocity of hyoid bone $(\mathrm{cm} / \mathrm{s})$ & $1.8 \pm 1.2$ & $2.6 \pm 1.4$ & $0.001 *$ \\
\hline Area of vallecula stasis $\left(\mathrm{cm}^{2}\right)$ & $0.9 \pm 0.6$ & $0.7 \pm 0.5$ & 0.070 \\
\hline Area of pyriform sinus stasis $\left(\mathrm{cm}^{2}\right)$ & $1.9 \pm 1.2$ & $1.3 \pm 0.9$ & 0.066 \\
\hline \multicolumn{4}{|l|}{$5 \mathrm{ml}$ paste $(n=10)$} \\
\hline Penetration-aspiration scale, score & $3.5 \pm 2.3$ & $3.0 \pm 2.6$ & 0.138 \\
\hline Oral transit time (s) & $4.1 \pm 3.8$ & $2.7 \pm 2.9$ & 0.059 \\
\hline Pharyngeal transit time (s) & $2.1 \pm 1.6$ & $2.0 \pm 1.8$ & 0.897 \\
\hline Pharyngeal delay time (s) & $0.6 \pm 0.8$ & $0.7 \pm 1.1$ & 0.746 \\
\hline Maximum displacement of hyoid bone $(\mathrm{cm})$ & $1.3 \pm 0.5$ & $1.2 \pm 0.6$ & 0.954 \\
\hline Forward displacement of hyoid bone $(\mathrm{cm})$ & $0.8 \pm 0.6$ & $0.8 \pm 0.6$ & 0.893 \\
\hline Upward displacement of hyoid bone (cm) & $0.8 \pm 0.5$ & $0.8 \pm 0.5$ & 0.962 \\
\hline Displacement duration of hyoid bone (s) & $0.8 \pm 0.4$ & $0.6 \pm 0.5$ & 0.220 \\
\hline Displacement speed of hyoid bone $(\mathrm{cm} / \mathrm{s})$ & $1.7 \pm 0.9$ & $2.4 \pm 1.5$ & $0.028 *$ \\
\hline Area of vallecula stasis $\left(\mathrm{cm}^{2}\right)$ & $1.2 \pm 0.6$ & $0.8 \pm 0.4$ & 0.194 \\
\hline Area of pyriform sinus stasis $\left(\mathrm{cm}^{2}\right)$ & $2.2 \pm 1.2$ & $1.4 \pm 0.8$ & $0.026^{*}$ \\
\hline
\end{tabular}

barium and from $1.7 \pm 0.9$ to $2.4 \pm 1.5 \mathrm{~cm} / \mathrm{s}(p=0.028)$ for the paste barium. No significant changes occurred in the stasis areas of the valleculae and pyriform sinus for the thin barium after therapy. However, those of the pyriform sinus for the paste bolus decreased significantly from $2.2 \pm 1.2$ to $1.4 \pm 0.8 \mathrm{~cm}^{2}(p=0.026$; Table 3).

Comparisons of the HRP group before and after therapy

The MDADI of the HRP group showed no significant changes after therapy. However, the PAS in swallowing the thin and the paste barium both increased: from $2.6 \pm 2.5$ to $4.2 \pm 2.4$ points $(p=0.061)$ for the thin barium and from $2.6 \pm 1.9$ to $4.6 \pm 2.7$ points $(p=0.037)$ for the paste barium. The displacements of the hyoid bone showed no significant changes for the thin barium. Anterior displacement decreased significantly from $1.1 \pm 0.7$ to $0.6 \pm 0.4 \mathrm{~cm}$ $(p=0.039)$, but superior displacement increased from $0.9 \pm$ 0.7 to $1.4 \pm 0.6 \mathrm{~cm}(p=0.041)$ when swallowing paste barium. The velocity of the hyoid bone and the stasis areas of the valleculae and pyriform sinus were not significantly different before and after therapy, either for the thin or the paste barium (Table 4).
Comparisons of changes after therapy

The PAS improvement of the FES group was significantly greater than that of the HRP group for swallowing the paste barium ( $p=0.016$ ). The velocity increase of the hyoid bone in the FES group was greater than the HRP group when swallowing the thin barium $(p<0.001)$, but no significant differences were found for temporal measures, displacements of the hyoid bone, the MDADI score, or bolus stasis (Table 5).

\section{Discussion}

In this study, FES therapy was found to improve the moving speed of the hyoid bone, the area of pyriform sinus stasis, and quality of life of NPC patients who suffered from dysphagia arising from RT. Satisfaction of treatment is a subjective feeling. For instance, an improved result could relate to the subject's simply receiving more attention from significant others rather than - in this study for instanceimprovement in their quality of life because of restored swallowing function. Nevertheless, the QoL score follows 
Table 4 Pre- and post-treatment in HRP group
$* p<0.05$, paired $t$ test

\begin{tabular}{|c|c|c|c|}
\hline Measurements & Pre-, mean $\pm \mathrm{SD}$ & Post-, mean \pm SD & $p$ \\
\hline $\begin{array}{l}\text { M.D. Anderson Dysphagia Inventory, total score } \\
5 \mathrm{ml} \text { thin }(n=10)\end{array}$ & $57.5 \pm 11.3$ & $58.0 \pm 10.4$ & 0.882 \\
\hline Penetration-aspiration scale, score & $2.6 \pm 2.5$ & $4.2 \pm 2.4$ & 0.061 \\
\hline Oral transit time (s) & $1.2 \pm 1.9$ & $0.9 \pm 1.3$ & 0.252 \\
\hline Pharyngeal transit time (s) & $2.8 \pm 3.5$ & $3.4 \pm 3.7$ & 0.553 \\
\hline Pharyngeal delay time (s) & $1.7 \pm 3.1$ & $2.7 \pm 3.8$ & 0.210 \\
\hline Maximum displacement of hyoid bone $(\mathrm{cm})$ & $1.3 \pm 0.4$ & $1.5 \pm 0.5$ & 0.303 \\
\hline Forward displacement of hyoid bone $(\mathrm{cm})$ & $0.9 \pm 0.5$ & $0.7 \pm 0.3$ & 0.372 \\
\hline Upward displacement of hyoid bone $(\mathrm{cm})$ & $0.7 \pm 0.6$ & $1.1 \pm 0.7$ & 0.116 \\
\hline Displacement duration of hyoid bone (s) & $0.7 \pm 0.3$ & $1.1 \pm 0.6$ & $0.032 *$ \\
\hline Displacement velocity of hyoid bone $(\mathrm{cm} / \mathrm{s})$ & $1.9 \pm 0.7$ & $1.5 \pm 0.6$ & 0.135 \\
\hline Area of vallecula stasis $\left(\mathrm{cm}^{2}\right)$ & $0.8 \pm 0.5$ & $0.8 \pm 0.4$ & 0.847 \\
\hline Area of pyriform sinus stasis $\left(\mathrm{cm}^{2}\right)$ & $1.8 \pm 0.7$ & $1.6 \pm 0.6$ & 0.331 \\
\hline \multicolumn{4}{|l|}{$5 \mathrm{ml}$ paste $(n=9)$} \\
\hline Penetration-aspiration scale, score & $2.6 \pm 1.9$ & $4.6 \pm 2.7$ & $0.037^{*}$ \\
\hline Oral transit time $(\mathrm{s})$ & $3.1 \pm 2.5$ & $1.9 \pm 1.9$ & 0.107 \\
\hline Pharyngeal transit time (s) & $2.8 \pm 2.8$ & $2.9 \pm 2.7$ & 0.918 \\
\hline Pharyngeal delay time (s) & $0.9 \pm 1.9$ & $1.4 \pm 2.2$ & 0.464 \\
\hline Maximum displacement of hyoid bone $(\mathrm{cm})$ & $1.7 \pm 0.5$ & $1.6 \pm 0.5$ & 0.732 \\
\hline Forward displacement of hyoid bone $(\mathrm{cm})$ & $1.1 \pm 0.7$ & $0.6 \pm 0.4$ & $0.039^{*}$ \\
\hline Upward displacement of hyoid bone $(\mathrm{cm})$ & $0.9 \pm 0.7$ & $1.4 \pm 0.6$ & $0.041^{*}$ \\
\hline Displacement duration of hyoid bone (s) & $0.9 \pm 0.5$ & $1.3 \pm 0.9$ & 0.343 \\
\hline Displacement speed of hyoid bone $(\mathrm{cm} / \mathrm{s})$ & $2.1 \pm 1.0$ & $1.8 \pm 1.4$ & 0.593 \\
\hline Area of vallecula stasis $\left(\mathrm{cm}^{2}\right)$ & $1.1 \pm 0.7$ & $1.0 \pm 0.5$ & 0.680 \\
\hline Area of pyriform sinus stasis $\left(\mathrm{cm}^{2}\right)$ & $1.9 \pm 1.0$ & $1.5 \pm 0.6$ & 0.400 \\
\hline
\end{tabular}

objective scoring to assess subjective feeling, and the improvement in QoL scores in this study was also supported by improvements under the other swallowing parameters. Since QoL is of key importance in clinical practice and useful for indicating the impact of dysphagia [23], we included QoL as an evaluation parameter in this study.

The decrease in stasis in the pyriform sinuses in the FES group following treatment indicates that the FES patients were able to swallow the bolus of food more easily, especially the paste bolus. Studies have found that NPC patients have difficulty in swallowing paste bolus because of fibrosis of their UES and that CP muscle resection is necessary to improve this condition [6]. In our study, the amount of bolus stasis at the pyriform sinuses following FES decreased significantly, and therefore, the amount of swallowed bolus increased significantly. This indicates greater ease of swallowing.

Following FES therapy, the patients were able to move the hyoid bone from its resting position to the highest position significantly faster than before. While no study to our knowledge has yet investigated the relationship between hyoid bone movement velocity and swallowing function, it is well known that elevation of the hyoid bone is important for closing the airway to prevent choking [26] and that elevation must be quick and timely to effectively protect the airway [27]. We therefore suggest that in this study, the increased velocity of the hyoid bone movement following FES helped to protect the airway and reduce aspiration.

The FES subjects' PAS dropped significantly from 3.8 to 2.9 , indicating that aspiration did decrease as a result of FES. Previous studies have shown that electrical currents can permanently change the characteristics of muscles after training at least 15 times [28-30] and concurrently increase the velocity of muscle contraction $[31,32]$. We also found that those FES subjects in our study who had developed muscle fibrosis following RT likewise experienced an increase in the velocity of their remaining muscles following FES. However, more detailed physiological examinations are necessary to confirm the mechanisms of this change.

The area of the paste bolus stasis at the NPC patients' pyriform sinuses decreased following FES. Pyriform sinus stasis results from abnormality in UES function and from insufficient anterior displacement of the hyoid bone. This leads not only to food sticking in the throat but also to 
Table 5 Comparison of the differences between pre- and post-treatment
${ }^{*} p<0.05 ; * * p<0.001$, paired $t$ test

\begin{tabular}{|c|c|c|c|}
\hline Measurements & $\mathrm{FES}$, mean $\pm \mathrm{SD}$ & $\mathrm{HRP}$, mean $\pm \mathrm{SD}$ & $p$ \\
\hline $\begin{array}{l}\text { M.D. Anderson Dysphagia Inventory, total score } \\
5 \mathrm{ml} \text { thin }(n=10)\end{array}$ & \multicolumn{2}{|c|}{$5 \mathrm{ml}$ thin $(n=10)$} & 0.053 \\
\hline Penetration-aspiration scale, score & $-0.9 \pm 2.3$ & $1.6 \pm 2.4$ & 0.103 \\
\hline Oral transit time (s) & $-0.3 \pm 0.8$ & $-0.3 \pm 0.8$ & 0.934 \\
\hline Pharyngeal transit time $(\mathrm{s})$ & $1.5 \pm 4.3$ & $0.6 \pm 3.1$ & 0.550 \\
\hline Pharyngeal delay time (s) & $-0.1 \pm 1.2$ & $0.9 \pm 2.2$ & 0.165 \\
\hline Maximum displacement of hyoid bone $(\mathrm{cm})$ & $0.1 \pm 0.4$ & $0.1 \pm 0.4$ & 0.622 \\
\hline Forward displacement of hyoid bone (cm) & $0.1 \pm 0.5$ & $-0.2 \pm 0.5$ & 0.249 \\
\hline Upward displacement of hyoid bone (cm) & $0.0 \pm 0.5$ & $0.4 \pm 0.8$ & 0.193 \\
\hline Duration of displacement of hyoid bone (s) & $-0.4 \pm 0.4$ & $0.4 \pm 0.4$ & $0.001 *$ \\
\hline Velocity of displacement of hyoid bone $(\mathrm{cm} / \mathrm{s})$ & $0.8 \pm 0.5$ & $-0.4 \pm 0.8$ & $0.001 * *$ \\
\hline Area of vallecula stasis $\left(\mathrm{cm}^{2}\right)$ & $-0.3 \pm 0.4$ & $0.0 \pm 0.6$ & 0.215 \\
\hline Area of pyriform sinus stasis $\left(\mathrm{cm}^{2}\right)$ & $-0.6 \pm 0.9$ & $-0.2 \pm 0.6$ & 0.311 \\
\hline \multicolumn{4}{|l|}{$5 \mathrm{ml}$ paste $(n=9)$} \\
\hline Penetration-aspiration scale, score & $-0.6 \pm 1.0$ & $2.0 \pm 2.4$ & $0.016^{*}$ \\
\hline Oral transit time $(\mathrm{s})$ & $-1.4 \pm 2.1$ & $-1.2 \pm 2.0$ & 0.815 \\
\hline Pharyngeal transit time (s) & $-0.4 \pm 2.4$ & $0.1 \pm 2.9$ & 0.719 \\
\hline Pharyngeal delay time (s) & $0.0 \pm 1.4$ & $0.4 \pm 1.7$ & 0.591 \\
\hline Maximum displacement of hyoid bone $(\mathrm{cm})$ & $0.1 \pm 0.5$ & $-0.1 \pm 0.6$ & 0.455 \\
\hline Forward displacement of hyoid bone $(\mathrm{cm})$ & $0.0 \pm 0.3$ & $-0.5 \pm 0.6$ & 0.059 \\
\hline Upward displacement of hyoid bone $(\mathrm{cm})$ & $0.2 \pm 0.5$ & $0.5 \pm 0.6$ & 0.236 \\
\hline Displacement duration of hyoid bone (s) & $-0.1 \pm 0.5$ & $0.4 \pm 1.1$ & 0.190 \\
\hline Displacement speed of hyoid bone $(\mathrm{cm} / \mathrm{s})$ & $0.8 \pm 0.8$ & $-0.3 \pm 1.8$ & 0.119 \\
\hline Area of vallecula stasis $\left(\mathrm{cm}^{2}\right)$ & $-0.3 \pm 0.9$ & $-0.1 \pm 0.6$ & 0.536 \\
\hline Area of pyriform sinus stasis $\left(\mathrm{cm}^{2}\right)$ & $-0.8 \pm 1.0$ & $-0.4 \pm 1.3$ & 0.295 \\
\hline
\end{tabular}

aspiration [2, 4-6]. Localized muscle fibrosis in NPC patients may alter the characteristics of the original muscle, resulting in a reduction in the anterior displacement of the hyoid bone and a lack of UES relaxation [4-6]. Following FES, although the anterior displacement of the hyoid bone in the supra-hyoid muscle group did not change, the hyoid bone velocity did increase significantly. This suggests that the efficiency of muscle contraction improved. This may be the reason for the reduction in the area of pyriform sinus stasis. Fibrosis of the CP muscle may also cause pyriform sinus stasis to increase. Further investigation into the pressure of the pharynx and CP muscle during swallowing by manometer would be worthwhile to explore this.

In this study, the patients who carried out the HRP experienced no improvement in their swallowing function [12]. Dysphagia in NPC patients principally occurs because fibrosis following RT leaves them with insufficient muscle strength to swallow. The positive effects reported in most previous studies on swallowing training in patients with NPC have been based on compensatory posture adjustments and alterations to bolus characteristics [8-11]. This study did not include compensatory posture adjustment techniques since it aimed to explore different techniques for strengthening muscles themselves as a more effective and substantial treatment to reduce dysphagia in post-RT NPC patients and improve the swallowing function aspect of their quality of life. The exercises in this study focused on strengthening the tongue, and pharyngeal muscles by repeated and effortful exercise. The HRP patients were telephoned every 2 weeks for encouragement and to keep on track with following the exercises. The results show that traditional muscle strengthening does not improve the swallowing function of NPC patients with post-RT dysphagia. Nevertheless, given the home setting of this activity, it was not possible to verify how regularly the patients adhered to it. Therefore, further study with hospital-based swallowing training programs or home training programs undertaken in the presence of a therapist could guarantee the compliance of the subjects and better trace the effects of swallowing muscle training.

When comparing the effects of the two different therapy methods on swallowing function, the FES group showed significantly more improvement than the HRP group in the PAS and velocity of the hyoid bone, but no significant difference was found in the displacement of the hyoid bone. We hypothesized that both FES and HRP may lead to the hypertrophy of the supra-hyoid muscle group, thus helping 
increase the elevation of the hyoid bone. However, our findings did not support this. Since patients with NPC generally have a reduced number of normal muscle fibers, further study is needed to evaluate whether 15 sessions of FES are sufficient to produce muscle hypertrophy [21].

Our team used quantitative assessment to objectively evaluate the effects of swallowing training. This included hyoid bone displacement and the status of stasis in the valleculae and pyriform sinuses. Although VFSS is the standard procedure for assessing swallowing function, it is still semiquantitative. The VFSS examination can quantitatively assess changes to transit time, but the assessment of the amount of stasis and larynx elevation is still qualitative. For this study, we used simple analysis tools that enabled changes of the variables relating to swallowing function to be assessed with very good reliability and which can be used in future swallowing function assessments.

By performing quantitative assessment of FES, we demonstrated that FES may increase the velocity of hyoid bone movement and reduce the pyriform sinus stasis. There were several limitations in this study. Firstly, the lack of information about individual variations to muscle fibrosis levels could have affected the results, although the mean DOSS scores were similar in both groups. Secondly, there was no control group. Nevertheless, although the swallowing function of the HRP group deteriorated over 3 months, a control group of patients who had undergone no treatment at all might have experienced greater deterioration than the HRP group in their swallowing function. Thirdly, the results from the small amount of subjects of the study may be an inaccurate reflection of the real and possible effects of FES. Therefore, results that were statistically insignificant for this study might be significant for a larger group.

A double-blinded clinical trial with a randomization design, an untreated control group, electromyography, and ultrasound imaging for more detailed data on relevant physiological mechanisms, would further clarify the effects of FES on dysphagia in NPC patients. Nevertheless, we are confident that our results form a solid basis for further investigations into this promising technique.

Acknowledgment We would like to thank the National Science Council Taiwan ROC for their support in funding this study and SLP (Meng-Chou Lee) for his assistance in collecting data.

\section{References}

1. Parkin DM, Whelan SL, Ferlay J et al (1997) Cancer incidence in five continents, vol 7. International Agency for Research on Cancer, Lyon, pp 334-337

2. Ku PK, Yuen EH, Cheung DM et al (2007) Early swallowing problems in a cohort of patients with nasopharyngeal carcinoma: symptomatology and videofluoroscopic findings. Laryngoscope 117:142-146
3. Lovell SJ, Wong HB, Loh KS et al (2005) Impact of dysphagia on quality-of-life in nasopharyngeal carcinoma. Head Neck 27:864-872

4. Eisbruch A, Lyden T, Bradford CR et al (2002) Objective assessment of swallowing dysfunction and aspiration after radiation concurrent with chemotherapy for head-and-neck cancer. Int J Radiat Oncol Biol Phys 53:23-28

5. Chang YC, Chen SY, Lui LT et al (2003) Dysphagia in patients with nasopharyngeal cancer after radiation therapy: a videofluoroscopic swallowing study. Dysphagia 18:135-143

6. Wu CH, Hsiao TY, Ko JY et al (2000) Dysphagia after radiotherapy: endoscopic examination of swallowing in patients with nasopharyngeal carcinoma. Ann Otol Rhinol Laryngol 109:320-325

7. Ishida R, Palmer JB, Hiiemae KM (2002) Hyoid motion during swallowing: factors affecting forward and upward displacement. Dysphagia 17:262-272

8. Lazarus C, Logemann JA, Gibbons P (1993) Effects of maneuvers on swallowing function in a dysphagic oral cancer patient. Head Neck 15:419-424

9. Lazarus CL, Logemann JA, Kahrilas PJ et al (1994) Swallow recovery in an oral cancer patient following surgery, radiotherapy, and hyperthermia. Head Neck 16:259-265

10. Logemann JA, Rademaker AW, Pauloski BR et al (1994) Effects of postural change on aspiration in head and neck surgical patients. Otolaryngol Head Neck Surg 110:222-227

11. Logemann JA, Pauloski BR, Rademaker AW et al (1997) Supersupraglottic swallow in irradiated head and neck cancer patients. Head Neck 19:535-540

12. Rosenthal DI, Lewin JS, Eisbruch A (2006) Prevention and treatment of dysphagia and aspiration after chemoradiation for head and neck cancer. J Clin Oncol 24:2636-2643

13. Carroll WR, Locher JL, Canon CL et al (2008) Pretreatment swallowing exercises improve swallow function after chemoradiation. Laryngoscope 118:39-43

14. Park CL, O’Neill PA, Martin DF (1997) A pilot exploratory study of oral electrical stimulation on swallow function following stroke: an innovative technique. Dysphagia 12:161-166

15. Freed ML, Freed L, Chatburn RL et al (2001) Electrical stimulation for swallowing disorders caused by stroke. Respiratory Care 46:466474

16. Leelamanit V, Limsakul C, Geater A (2002) Synchronized electrical stimulation in treating pharyngeal dysphagia. Laryngoscope 112:2204-2210

17. Blumenfeld L, Hahn Y, LePage A et al (2006) Transcutaneous electrical stimulation versus traditional dysphagia therapy: a nonconcurrent cohort study. Otolaryngol Head Neck Surg 135:754-757

18. Carnaby-Mann GD, Crary MA (2007) Examining the evidence on neuro-muscular electrical stimulation for swallowing: a meta-analysis. Arch Otolaryngol Head Neck Surg 133:564-571

19. Shaw GY, Sechtem PR, Searl J et al (2007) Transcutaneous neuromuscular electrical stimulation (VitalStim) curative therapy for severe dysphagia: myth or reality? Ann Otol Rhinol Laryngol 116:36-44

20. Bülow M, Speyer R, Baijens L et al (2008) Neuromuscular electrical stimulation (NMES) in stroke patients with oral and pharyngeal dysfunction. Dysphagia 23:302-309

21. Carnaby-Mann GD, Crary MA (2008) Adjunctive neuromuscular electrical stimulation for treatment-refractory dysphagia. Ann Otol Rhinol Laryngol 117:279-287

22. O'Neil KH, Purdy M, Falk J et al (1999) The dysphagia outcome and severity scale. Dysphagia 14:139-145

23. Chen AY, Frankowski R, Bishop-Leone J et al (2001) The development and validation of a dysphagia-specific quality-of-life questionnaire for patients with head and neck cancer. Arch Otolaryngol Head Neck Surg 127:870-876 
24. Rosenbek JC, Robbins JA, Roecker EB et al (1996) A penetration-aspiration scale. Dysphagia 11:93-98

25. Logemann JA (1998) Evaluation and treatment of swallowing disorders, 2nd edn. Pro-Ed, Texas, pp 91-109

26. Kim Y, McCullough GH (2008) Maximum hyoid displacement in normal swallowing. Dysphagia 23:274-279

27. Kendall KA, Leonard RJ (2001) Hyoid movement during swallowing in older patients with dysphagia. Arch Otolaryngol Head Neck Surg 127:1224-1229

28. Hudlická O, Brown M, Cotter M et al (1977) The effect of long-term stimulation of fast muscles on their blood flow, metabolism and ability to withstand fatigue. Pflügers Arch 369:141149
29. Pette D, Müller W, Leisner E et al (1976) Time dependent effects on contractile properties, fibre population, myosin light chains and enzymes of energy metabolism in intermittently and continuously stimulated fast twitch muscles of the rabbit. Pflügers Arch 364:103-112

30. Salmons S, Sréter FA (1976) Significance of impulse activity in the transformation of skeletal muscle type. Nature 263:30-34

31. Hennig R, Lømo T (1987) Effects of chronic stimulation on the size and speed of long-term denervated and innervated rat fast and slow skeletal muscles. Acta Physiol Scand 130:115-131

32. Karba R, Stefanovska A, Dordević S (1990) Human skeletal muscle: phasic type of electrical stimulation increases its contractile speed. Ann Biomed Eng 18:479-490 